\title{
GRUPOS DE APOYO PARA ESTUDIANTES \\ UNIVERSITARIOS GAY, LESBIANAS Y BISEXUALES
}

\author{
Mabel T. López Ortiz ${ }^{1}$
}

\section{Resumen}

Este Artículo discute la evaluación del Modelo de Grupo de Apoyo para Estudiantes: Gays Lesbianas y Bisexuales del Departamento de Consejería para el Desarrollo Estudiantil de la Universidad de Puerto Rico, Recinto de Río Piedras. La muestra contó con estudiantes que participaron en un grupo antes del año académico 2006-2007. Los objetivos de la evaluación eran corroborar la percepción de satisfacción de los estudiantes con la metodología grupal así como conocer sus recomendaciones para la vigencia y continuidad del grupo. Desde una perspectiva ecológica se analizan las situaciones que rodean a este grupo de estudiantes como parte de una sociedad homofóbica y heterosexista y que fundamentan que el grupo permanezca desde su fundación en el 1989 hasta el presente. Los hallazgos revelan un $100 \%$ de satisfacción de la muestra con la modalidad de grupo de apoyo lo que confirma la percepción sobre la vigencia del grupo. También mostraron valorar el rol de las facilitadoras y de sus pares en el grupo así como la recomendación de que este grupo continúe como parte de los servicios del Departamento de Consejería para el Desarrollo Estudiantil.

Descriptores: Grupo de Apoyo, Estudiantes Gays, Lesbianas y Bisexuales.

${ }^{1}$ Profesora en el Departamento de Trabajo Social, Facultad de Ciencias Sociales, Universidad de Puerto Rico en Río Piedras. 


\section{Abstract}

This article discusses the evaluation of the Model Support Group for Students: Gay Lesbian and Bisexual of the Department of Counseling for Student Development at the University of Puerto Rico at Rio Piedras Campus. The sample were students who participated in a group prior to the academic year 2006-2007. The objectives of the evaluation were to corroborate the perception of satisfaction of students with the methodology group and explore their recommendations for the continuity of the group. From an ecological perspective analyzes the situations surrounding this group of students as part of a society homophobic and heterosexist and that the group remains based since its founding in 1989 until the present. The findings reveal a $100 \%$ satisfaction of the sample with the support group, which confirms the perception about the validity of the group. It also showed assess the role of facilitators and their peers in the group as well as the recommendation that this group will continue as part of the services of the Department of Counseling for the Student Development.

Key words: Support Group, Students Gays, Lesbians and Bisexuals.

\section{Introducción}

El Departamento de Consejería para el Desarrollo Estudiantil del Decanato de Estudiantes del Recinto de Río Piedras fue pionero en crear un grupo de apoyo dirigido a estudiantes gays, lesbianas y bisexuales (GLB) por iniciativa de Carlos Dalmau, trabajador social y Elena De Jesús, consejera. Todos los semestres, desde el 1989 hasta el presente se reúnen como grupo, estudiantes diversos con una característica en común: no reconocerse heterosexual. Este es el único grupo conocido dirigido a estudiantes GLB en el sistema universitario público y privado de nuestro país.

Durante varios semestres las personas organizadoras del grupo de apoyo GLB pudieron identificar entre el estudiantado que solicitaba servicio, la necesidad de este grupo de jóvenes de estar en un ambiente de aceptación, confidencialidad y apoyo que a su vez les ayudara en la consecución de su grado universitario. Esta población, que puede ser marginada en la familia $y$ en la sociedad en general, también enfrentaba situaciones de rechazo en 
el ámbito universitario. Nuestras universidades como parte de la sociedad son micro estructuras que no están al margen del ambiente homofóbico en Puerto Rico. Esta situación originó la atención inmediata del equipo multidisciplinario que se trazó el propósito y objetivo de contribuir a crear un balance en el desarrollo emocional y académico de este grupo de estudiantes a través de la discusión de situaciones relacionadas con su orientación. En este proceso de interacción grupal los miembros aprenden y desarrollan destrezas que pueden facilitar la solución y manejo de situaciones del diario vivir.

Centrados en casos individuales se forma el primer grupo de apoyo para estudiantes Gay, Lesbianas y Bisexuales de nuestro Recinto. A través de los años el grupo se ha mantenido con la experiencia de varias personas como facilitadoras entre éstas la autora de este artículo como trabajadora social y Elena de Jesús Graham, como consejera, organizadora y colíder del grupo. La necesidad y vigencia de este grupo único en las Universidades públicas y privadas de Puerto Rico, fueron los ingredientes necesarios para motivar a ambas colíderes a explorar y conocer la percepción sobre la experiencia grupal de personas ex miembros del grupo a través de este ejercicio de investigación.

\section{Grupos de Apoyo GLB}

Los grupos de apoyo como metodología o modalidad de ayuda trabajan con preocupaciones, necesidades o situaciones en común que caracterizan o comparten un grupo de personas. Los beneficios de esta modalidad de grupo son discutidos a través de la literatura en el campo de las profesiones de ayuda que buscan trabajar efectivamente con diferentes poblaciones como parte de la diversidad humana (De Vidas, 1999; Robinson, 1994). Esta modalidad fue utilizada para trabajar con estudiantes universitarios gay, lesbianas y bisexuales. La característica en común, del grupo de estudiantes - la diversidad en su orientación sexual- en una sociedad heterosexista y homofóbica puede apuntar a dificultades con consecuencias únicas y posiblemente nefastas para este grupo de jóvenes. Como señalan Toro-Alfonso y Varas (2004:548): "La manifestación de rechazo y prejuicio contra la homosexualidad y el lesbianismo atenta brutalmente sobre la seguridad física y emocional de la juventud que está en el proceso de enfrentarse a su orientación sexual." Los autores citados realizaron un ejercicio de comparar estudios sobre el prejuicio y el discrimen hacia las 
personas gays en el cual develaron que el prejuicio y el rechazo social contra gays y lesbianas son comunes entre estudiantes universitarios.

Con este escenario comienza el proceso de ajuste a la vida universitaria de cada joven GLB que inicia una formación profesional. Para este grupo de estudiantes se suma el manejo de situaciones relacionadas con su orientación sexual como lo son: el proceso de salir del clóset, discrimen institucional, homofobia, rechazo familiar, aislamiento, invisibilidad, anonimato y exclusión de la comunidad universitaria. Toro-Alfonso y Varas (2004:538) confirman que: "Los gays y lesbianas representan un sector de nuestra sociedad que es rechazado y enfrenta sanciones morales, religiosas y legales." Frente a esta realidad y a las necesidades de este grupo de estudiantes surge la motivación de la creación del Grupo de Apoyo como respuesta afirmativa de profesionales del Departamento de Consejería para el Desarrollo Estudiantil, el cual se mantiene al presente con la apreciación de obtener beneficios de ayuda para estudiantes gays, lesbianas y bisexuales dentro del ambiente universitario.

El grupo de apoyo representa un mensaje de aceptación para esta juventud que enfrenta múltiples situaciones que se generan en una sociedad que no maneja efectivamente la diversidad. De Vidas (1999:52) indica que: "La formación de estos tipos de grupos ayudan a adoptar la auto aceptación y afirmación de la identidad homosexual, lésbica y bisexual y reduce el sentido de marginación y aislamiento de los miembros". Se suma al ambiente de aceptación, la discusión abierta de la orientación sexual del equipo de facilitadores los cuales hasta el presente han sido heterosexuales. El trabajo de profesionales heterosexuales envía un mensaje directo contra la homofobia y puede generar vínculos o alianzas para una lucha unida. Según Chojnacki y Gelberg (1995:354):

Sólo a través del envolvimiento de la población heterosexual en situaciones de la comunidad GLB es que se puede atender directa y completamente la homofobia y el heterosexismo, y así, mejorar la calidad de los servicios terapéuticos ofrecidos a la comunidad GLB (pág. 354).

Desde sus comienzos hasta el presente el Grupo de Apoyo GLB se reúne semestralmente con el apoyo de dos personas facilitadoras. La participación del estudiantado es voluntaria y requiere de una entrevista individual previa a participar en la dinámica grupal. En esta entrevista se 
discuten la identidad sexual de cada estudiante (como requisito del Grupo debes reconocerte homosexual, lesbiana o bisexual) y los propósitos y objetivos del grupo (se ofrecen por escrito) así como unas normas generales en términos de un contrato inicial.

Entre estas normas se puntualiza sobre la confidencialidad del día, hora y lugar de reunión, la participación voluntaria y la integración al grupo a través de las preguntas generadas por sus miembros en la reunión inicial. Las personas facilitadoras ponderan en esa entrevista inicial si la persona interesada está preparada para la dinámica grupal. Algunas situaciones ameritan ayuda individual antes de comenzar el grupo como por ejemplo confusión en la identidad sexual, características de mucha tristeza o ideas suicidas, entre otras. Con una estructura flexible la dinámica grupal se inicia con la presentación de nuevos miembros y la interacción con miembros activos, luego se discuten las situaciones que el grupo quiera discutir tanto individuales como grupales. A través de la dinámica grupal sus miembros ofrecen consejos guía y modelaje de solucionar problemas de la vida cotidiana relacionados con la orientación sexual. Como norma y regla grupal se integran recursos de la comunidad para discutir temas de interés de todas las personas y una vez al año se realiza una actividad masiva para la comunidad universitaria como Cine Foro, Feria de Servicios y otras actividades que respeten y cumplan con las normas del grupo. El ambiente grupal es participativo y de respeto a la diversidad entre sus miembros y facilitadoras.

\section{Perspectiva Ecológica y Organización de Grupos de Apoyo}

La perspectiva ecológica o de persona en su ambiente permite analizar cómo afecta a la juventud homosexual, lesbiana o bisexual el contexto o entorno de una sociedad heterosexista y homofóbica. Las demandas que exige un mundo donde los mensajes y modelos giran en torno a una sola orientación sexual (heterosexual) sin aceptar la diversidad, generan necesidades y situaciones particulares en las personas gays y bisexuales. Según Mallon (1998) como persona gay lesbiana o bisexual luchas por corresponder de la mejor forma con el ambiente, pero puedes encontrar la intolerancia del ambiente y que el ajuste no es bueno. La juventud GLB tiene que realizar ejercicios mentales constantes para manejar su conducta en diferentes escenarios y evaluar las consecuencias de salir o no del clóset o armario sin la posibilidad de tener alternativas de modelos GLB para el 
manejo de estas realidades. Cada joven busca responder adecuadamente a las demandas del entorno, cuando esto no ocurre pueden surgir el estrés, los sentimientos de culpa, problemas emocionales, grados de impotencia, problemas de autoestima y la homofobia internalizada que lo aquejan. Utilizar el modelo ecológico como acercamiento o perspectiva teórica o conceptual permite que podamos ver las dificultades experimentadas por la juventud homosexual, lésbica y bisexual. Estas dificultades se interpretan como las manifestaciones de pobre ajuste o ausencia de interacción entre cada joven y su ambiente (Elze, 2001:98).

Las necesidades y realidades particulares de esta población así como la concienciación de una determinación inclusiva requieren de una base de conocimientos del tema. El estudiantado gay, lésbico y bisexual en la mayoría de los casos carece o tiene sistemas de apoyo limitados a su alrededor. Posiblemente vienen de familias heterosexistas con modelos y expectativas en la misma dirección lo cual crea una serie de presiones adicionales a las que enfrentan la juventud heterosexual. Por otro lado, durante la vida universitaria parte del estudiantado no cuenta con su familia y amistades cercanas por la distancia entre su pueblo de origen y la universidad, lo cual puede contribuir al desfase en su ajuste entre ambiente y persona, que en ocasiones les motiva a la búsqueda de ayuda profesional. A estas circunstancias se une un sector de jóvenes GLB, quienes tampoco se benefician de las funciones afectivas, sociales e instrumentales de su familia por razones a su orientación.

Es importante destacar la relación del nivel de salud mental familiar y la percepción de cada joven sobre la aceptación de su entorno. La apreciación cognitiva de eventos estresantes y de rechazo del entorno afecta la salud mental de cualquier joven. En un estudio citado por Van Wormer y McKinney (2003) se encontró que los conflictos que rodean la revelación de la orientación sexual gay, lesbiana y bisexual pueden influir en intentos suicidas en la juventud GLB (Friedman \& Downey, 1994).

A la situación de posibles conflictos y dificultades familiares se suman diversas situaciones de rechazo en los distintos escenarios en los que actúan la juventud GLB. En un estudio realizado en New England con adolescentes gay, lesbianas y bisexuales se encontró que compartían estresores con otras poblaciones vulnerables de adolescentes, pero que también enfrentaban retos psicológicos únicos por pertenecer a un grupo 
estigmatizado (Elze, 2001:96). Esto reitera la necesidad de intervención desde varias metodologías, en particular en términos de apoyo a malestares únicos de la población de estudiantes GLB de nuestra Universidad. La Asociación de Psicología Americana (APA) trazó unas guías para psicoterapia con clientes gays, lesbianas y bisexuales donde puntualizan las consecuencias de la estigmatización social (discriminación, prejuicios y violencia) en la salud mental y el bienestar de esta población.

En Puerto Rico en los últimos años hemos tenido cambios positivos para la población LGBTT $^{2}$, lo cual contribuye a los cambios en la percepción social de este grupo o minoría sexual. En el 2004 se eliminó del Código Penal el Artículo 103 el cual criminalizaba a las personas LGBTT en sus relaciones de pareja. Siguen aumentando las Iglesias de puertas abiertas para esta población y se discute en la Legislatura de PR la medida que incluye a las parejas de hecho en la definición de familias en el Código Civil aunque existen sectores de la comunidad que se oponen a toda medida que asegure derechos generales para las personas gay lesbianas y bisexuales. Todavía las parejas del mismo sexo carecen de protección legal en situaciones de violencia doméstica y derechos adscritos a la población heterosexual de nuestro país.

Desde una perspectiva ecológica debemos atender la relación entre el ambiente universitario, familiar y social y el funcionamiento académico y emocional del estudiantado GLB. La literatura sobre la población GLB sugiere una asociación entre las percepciones negativas de la comunidad joven LGTTB -como minoría sexual- con problemas sociales como la homofobia, rechazo, discrimen, entre otros.

Para lograr desarrollo integral de nuestros estudiantes se requieren respuestas institucionales o de programas de ayuda que puedan establecer el balance en las transacciones del estudiante y su ambiente. La profesión de trabajo social y consejería en escenarios universitarios, tienen como propósito lograr el funcionamiento y el ajuste a la vida universitaria de cada joven. Por esta razón el grupo de apoyo es una respuesta positiva a la filosofía y propósitos de estas profesiones y a las necesidades de este estudiantado. Elze (2001) asegura que tanto la profesión de trabajo social

${ }^{2}$ Las siglas LGBTT se refieren a personas lesbianas, gay, bisexuales, transexual y transgénero. 
como los grupos de apoyo pueden ofrecerle a la juventud gay, lésbica y bisexual oportunidades para terminar con el aislamiento, iniciar amistades, proveer de apoyo mutuo, desarrollar destrezas sociales y relaciones de pares saludables.

Para lograr el apoyo y una intervención sensible a esta minoría los profesionales de ayuda deben iniciar un camino hacia el conocimiento y el manejo de actitudes. Como López (2003) señala, “Aunque políticamente nuestra intervención sea la correcta basada en el conocimiento y los valores éticos, hay una dimensión que debemos confrontar: el nivel de homofobia y competencia". La intervención individual o grupal apropiada con jóvenes GLB, debe completarse cuando los profesionales comienzan con una reflexión de sus propias preocupaciones y sentimientos sobre el tema de la orientación sexual. Tanto la profesión de trabajo social como otras profesiones que trabajan con la persona y su ambiente caminamos en dirección del manejo de la diversidad humana como fortaleza de una intervención inclusiva.

Cada ser humano depende de otras personas. Toda persona es sostenida, en cierta medida, por el apoyo psicológico, emocional, espiritual y material de los demás. Según el Dr. Richard Pearson (1982) se han identificado trece tipos de apoyo personal que recibimos de los demás, entre ellos se encuentran la admiración, el compañerismo, el estímulo, la aceptación, el modelaje, la ayuda y la honestidad. El elemento común entre todas aquellas personas que constituyen lo que podría llamarse nuestro sistema de apoyo personal, es que nuestra relación con ellas, ya sea real o psicológica, puede contribuir a sentimientos positivos acerca de nuestra situación de vida y estima propia. Existen personas especiales cuya presencia constituye una fuerza positiva y enriquecedora en nuestras vidas, lo que podemos llamar una aportación a nuestras experiencias, pero la realidad es que la juventud GLB puede tener poco apoyo en una sociedad donde la familia, el vecino, la iglesia, las amistades, las instituciones sociales formales, pueden haber manifestado rechazo, intolerancia o, simplemente, invisibilizar la orientación sexual de la persona.

La evidencia científica confirma que las personas que cuentan con apoyo social, tienen mayores probabilidades de lograr un estado de salud óptimo. El apoyo social es un determinante en la salud y la ausencia de éste es un riesgo a la integridad de la persona (Toro-Alfonso, 2004). El Grupo de 
Apoyo GLB durante la vida universitaria puede aportar a llenar estos espacios vacíos. Los hallazgos de la evaluación del Modelo mostraron el apoyo que el grupo puede cumplir en la vida de esta población estudiantil y que tiene un alto valor para éstos (ver hallazgos).

La metodología grupal de apoyo es un modelo de intervención mutua y recíproca. Se reconoce como un tipo de grupo de ayuda, según Toseland y Rivas (2001), que se distingue por su propósito de fomentar la ayuda mutua entre sus miembros, facilitar a éstos el manejo de eventos o situaciones de vida conflictivas o estresantes, así como aumentar las habilidades y destrezas que les permitan adaptarse y manejar efectivamente futuras situaciones, conflictos o tensiones en su vida, a través de la dinámica grupal. Los grupos de apoyo se organizan para atender unas necesidades, características, situaciones o factores que sus miembros comparten en común y que les brindan una identidad colectiva.

El liderazgo (consejería, trabajo social, psicología y otras profesiones) en un grupo de apoyo tiene la perspectiva de facilitar que las personas compartan sus experiencias en el manejo de situaciones estresantes en sus vidas. Este puede ser de una persona o de dos: líder y colíder. La práctica del coliderazgo posee varias ventajas; como el obtener retroalimentación de las intervenciones, ofrecer modelos efectivos de comunicación, aportar a una filosofía democrática y compartir el poder en la toma de decisiones. Las personas líderes se convierten en facilitadores que propician una dinámica para que se logre el sistema de ayuda mutua. Las destrezas importantes en un grupo de esta índole son: el saber escuchar, capacidad para tomar decisiones democráticas, promover el trabajo en equipo y la participación voluntaria. Cuando hablamos de un grupo de apoyo dirigido a atender personas GLB, como facilitadores tenemos que autoevaluar nuestras actitudes y valores personales que puedan interferir en una intervención grupal inclusiva, justa y libre de prejuicios que defina practicas adecuadas de apoyo. Para algunos autores un rol importante del liderazgo en un grupo de apoyo es ofrecer esperanza y motivación dirigida al futuro a solucionar problemas, (Toseland \& Rivas, 2001:24).

Las técnicas de intervención grupal son no estructuradas y buscan promover la discusión y análisis de situaciones de vida así como compartir estrategias de manejo variadas entre sus miembros que sirvan de guías y modelos efectivos. En la atención de la filosofía de apoyo o asistencia, la 
intervención debe dirigirse a crear un ambiente de aceptación, respeto y confidencialidad para así facilitar la expresión de situaciones, ideas y sentimientos en el grupo. En este ambiente cada miembro siente que tiene una responsabilidad para con los demás en cuanto a ayudarlos en su crecimiento personal y al mismo tiempo siente que los demás pueden contribuir a su crecimiento como ser humano. Esto permite que se creen vínculos fuertes de afecto ya que sus miembros comparten su vida.

Aunque los grupos de apoyo pueden variar enormemente, todos comparten una algo en común, son espacios donde sus miembros pueden compartir historias personales, expresar sus emociones, ser escuchados en un ambiente de aceptación, entendimiento y empatía. Comparten información $\mathrm{y}$ recursos en la comunidad. Al ayudar a otros, las personas se fortalecen $\mathrm{y}$ capacitan a sí mismas. El grupo de apoyo puede servir de estímulo para que esta población se fortalezca a través de sentirse bien consigo misma en su totalidad como ser humano. Lograr insertarse en un ámbito social donde la persona pueda relacionarse y crear un círculo de amistades en el que se sienta aceptado es fundamental. El grupo se convierte en uno de los medios para salir del aislamiento, de sentimientos autodestructivos y reforzar la autoestima.

El Grupo de Apoyo con personas GLB puede facilitar una identidad positiva que aporte a un mejor estado de salud mental y una mayor autoestima. Entre las características de una autoestima positiva en una persona GLB está el creer firmemente en sus valores y principios y estar dispuesta a defenderlos aún cuando encuentre oposición de la sociedad, lo que se puede desarrollar a través de la dinámica del grupo. Es capaz de obrar según crea más acertado, confiando en su propio juicio sin sentirse culpable cuando a los demás miembros de la sociedad les parezca lo contrario. El grupo de apoyo a través de las experiencias compartidas puede tener el efecto de fomentar una autoestima apropiada, de reforzar el conjunto de sentimientos valorativos del "ser" de sus miembros, de manera integral incluyendo lo racional, espiritual, emocional y sexual. Esto también puede resultar del proceso de empoderamiento individual que puede ocurrir de la dinámica grupal.

Se ha discutido en varios foros la deseabilidad de que las personas facilitadores de un grupo de apoyo GLB sean gays o heterosexuales. En la 
situación de DCODE, las personas que han mostrado interés en participar en este tipo de grupo han sido heterosexuales, aunque no es el requisito.

Las primeras personas facilitadores del Grupo de Apoyo GLB de DCODE observaron a través de la experiencia grupal, que existía un paralelismo entre el proceso del estudiantado de salir del clóset y el proceso de ambos facilitadores de salir del clóset como profesionales de ayuda aliados al estudiante GLB. El crecimiento personal y profesional de los profesionales de ayuda heterosexuales se puede comparar con los modelos de formación de la identidad sexual los cuales, aunque están creados para el proceso de la persona GLB, las personas facilitadores heterosexuales pueden identificarse con las etapas discutidas en la literatura, en su proceso de empatía y compromiso con esta población. Tenemos la esperanza de que mayor cantidad de profesionales de ayuda sientan el compromiso de trabajar con una mentalidad libre de homofobia y heterosexismo, así se podrán ofrecer servicios de calidad a la diversidad sexual de nuestros estudiantes y aportar a un ambiente de aceptación.

Dentro de este contexto teórico y partiendo de la experiencia de más de dos décadas de desarrollo del grupo de apoyo, decidimos explorar diversas dimensiones de esta experiencia. En la próxima sección se describen los objetivos de la investigación.

\section{Metodología}

Con el propósito de mantener los servicios de apoyo a esta población a través del Grupo de Apoyo, trazamos los objetivos de: 1) evaluar el nivel de satisfacción (con la modalidad grupal, facilitadoras y pares) y los beneficios obtenidos de estudiantes que han participado del Grupo de Apoyo GLB de la Universidad de Puerto Rico, Recinto de Río Piedras, 2) explorar las razones expuestas por la muestra de estudiantes para la permanencia de este grupo, y 3 ) conocer sus recomendaciones.

Esta investigación de tipo exploratoria, inició con la aplicación de un cuestionario autoadministrable a una muestra voluntaria de jóvenes gays, lesbianas y bisexuales universitarios o graduados que participaron del grupo de apoyo en los años académicos previos al año académico 20062007. Las investigadoras trazaron un protocolo de investigación que cumplió con la autorización del Comité Institucional para la Protección de 
los Seres Humanos en la Investigación (CIPSHI) del Recinto de Río Piedras.

La selección de participantes se estableció por disponibilidad. Para contactar a las personas que participaron de este ejercicio se utilizaron los listados confidenciales que cada semestre organizan las personas que forman el grupo con números telefónicos y direcciones electrónicas, algunos de estos contactos ya no estaban activos. El primer contacto se realizó por vía telefónica o correo electrónico invitándoles a participar. Las personas que aceptaron participar recibieron físicamente o por correo electrónico (solicitado por la persona) el cuestionario y consentimiento informado manteniendo el principio de confidencialidad y anonimato de la persona encuestada.

Con el análisis de los hallazgos intentamos alcanzar el propósito de contribuir con información necesaria para el desarrollo de un modelo de grupo de apoyo para estudiantes universitarios gays, lesbianas y bisexuales como alternativa de ayuda.

El cuestionario incluyó cuatro partes con los temas a explorar: Perfil Sociodemográfico, Situaciones Personales, Grupo de Apoyo y Opinión de la muestra. Se utilizó una escala Likert con reactivos sobre el tema de Grupo de Apoyo y dos preguntas abiertas para obtener alguna información de tipo cualitativa sobre los objetivos descritos.

\section{Resumen de Hallazgos}

Perfil de la Muestra:

Del listado confidencial de participantes grupo resultó positivo el contacto con 41 personas, los números o correos restantes en los listados estaban incorrectos o inactivos. A pesar de la afirmación para participar de todas las personas contactadas del listado sólo contestaron el cuestionario 20, lo que representó un 50 por ciento de las personas invitadas a participar. El perfil demográfico de la muestra fue en su mayoría jóvenes gays masculinos, los cuales se encontraban en su tercer año de bachillerato. La facultad de más frecuencia fue Ciencias Sociales -aunque la muestra 
incluyó estudiantes de todas las facultades- y los años de participación en el grupo fueron alrededor de 3 años.

En el apartado de Situaciones Personales obtuvimos que sobre el manejo de su orientación sexual, esta muestra había compartido la noticia con otras personas, en su mayoría con amistades GLB (100\%). Entre los problemas enfrentados luego de reconocerse GLB sobresale la aceptación de la familia (65\%), los conflictos religiosos $(45 \%)$ y el proceso de salir del clóset (45\%), (ver Figura 1).

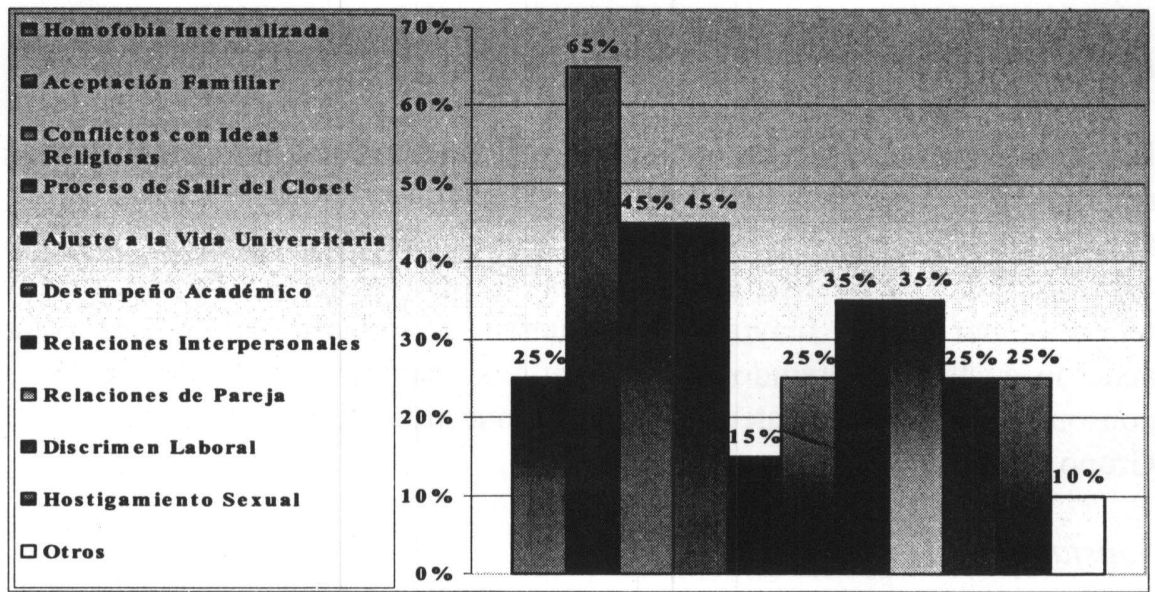

Figura 1. Problemas presentados luego reconocer la orientación sexual

Para enfrentar estos problemas un $80 \%$ señaló recibir ayuda del Grupo de Apoyo, 55\% del Departamento de Consejería para el Desarrollo Estudiantil, $50 \%$ y de amistades como los primeros sistemas de apoyo. Perciben como difícil la aceptación de los padres, el manejo de la homofobia internalizada, las relaciones de parejas y el proceso de salir del clóset. En estos momentos indican sentirse bien con su orientación sexual.

\section{Evaluación del Grupo de Apoyo}

Se realizaron preguntas dirigidas y abiertas para evaluar la percepción y opinión de la muestra de estudiantes sobre la experiencia de Grupo de Apoyo GLB. Se utilizó una escala Likert con 14 reactivos para contestar con los valores: acuerdo, imparcial y desacuerdo. Para analizar los datos 
obtenidos diferenciamos en las siguientes categorías: preferencia sobre la metodología de ayuda, beneficios obtenidos, satisfacción con el grupo, satisfacción con las facilitadoras y rol de los pares.

\section{Preferencia sobre metodología de ayuda}

Sobre la modalidad grupal de ayuda los estudiantes la prefieren en un $60 \%$, y el por ciento restante se mantuvo imparcial, de igual forma también un $60 \%$ afirmó preferir la modalidad individual.

\section{Beneficios Obtenidos}

Los reactivos que exploraron los beneficios de participar del Grupo se resumen en los temas de: reconocer situaciones similares entre pares, ayudar en situaciones relacionadas a la orientación, expresión libre sin temor a juicios, y facilitar el ajuste a la vida universitaria. En todos estos temas el por ciento mayor de la muestra indicó afirmativamente estar acuerdo en haber obtenido estos beneficios (ver tabla núm. 1). Esto es cónsono al hallazgo de que el $80 \%$ de la muestra recibió ayuda a través del Grupo de Apoyo.

\section{Satisfacción con el Grupo}

La satisfacción de la muestra con el grupo se midió a través de 4 reactivos sobre: los temas que se discuten, la satisfacción, información ofrecida, y recomendación sobre la permanencia del grupo como servicio. La tendencia en las respuestas fue a expresar satisfacción con los temas y preguntas (ver Tabla 2). Sobresale que $95 \%$ de la muestra expresó sentirse no juzgada durante su experiencia grupa 
Tabla Núm. 1

Beneficios Obtenidos

\begin{tabular}{llc}
\hline Categoría & $\begin{array}{l}\text { Premisa: El Grupo } \\
\text { de Apoyo... }\end{array}$ & De acuerdo \% \\
\hline $\begin{array}{l}\text { Beneficios } \\
\text { Obtenidos }\end{array}$ & $\begin{array}{l}\text { "me permitió ver que mi situación es } \\
\text { similar a la de otras personas" }\end{array}$ & $75 \%$ \\
& $\begin{array}{l}\text { "me ayudó en las situaciones } \\
\text { relacionadas a mi orientación" } \\
\text { "me permitió expresarme sin temor a } \\
\text { que me juzgaran" }\end{array}$ & $80 \%$ \\
& $\begin{array}{l}\text { "facilitó mi ajuste a la vida } \\
\text { universitaria" }\end{array}$ & 70 \\
\hline
\end{tabular}

Satisfacción con las Facilitadoras

La satisfacción con el rol de las facilitadoras en el grupo fue evaluada positivamente o en acuerdo por la muestra con dos reactivos que se relacionan con el apoyo ( $85 \%)$ y el respeto $(100 \%)$, ofrecido (ver Tabla 2 ).

\begin{tabular}{|c|c|c|}
\hline $\begin{array}{l}\text { Satisfacción } \\
\text { con: }\end{array}$ & Premisas: & $\begin{array}{c}\text { De acuerdo } \\
\%\end{array}$ \\
\hline \multirow[t]{4}{*}{ Grupo } & $\begin{array}{l}\text { "los temas que se discutían eran de } \\
\text { mi interés" }\end{array}$ & $85 \%$ \\
\hline & $\begin{array}{l}\text { "estoy satisfech@ con la ayuda del } \\
\text { grupo" }\end{array}$ & $95 \%$ \\
\hline & $\begin{array}{l}\text { "el grupo me facilitó información } \\
\text { sobre los procesos relacionados con } \\
\text { mi orientación sexual" }\end{array}$ & $90 \%$ \\
\hline & $\begin{array}{l}\text { "opino que el grupo debe } \\
\text { permanecer como modalidad de } \\
\text { ayuda al estudiante en la UPR" }\end{array}$ & 100 \\
\hline \multirow[t]{2}{*}{ Facilitadoras } & $\begin{array}{l}\text { "me ofrecieron el apoyo que } \\
\text { necesitaba" }\end{array}$ & $85 \%$ \\
\hline & $\begin{array}{l}\text { "mostraron respeto a las personas } \\
\text { participantes del grupo" }\end{array}$ & $100 \%$ \\
\hline
\end{tabular}

Rol de los Pares 
Sobre el rol de los pares se utilizaron dos premisas una con valor positivo y otra con uno negativo. La muestra se mantuvo distribuida de forma distinta que en los reactivos anteriores, aunque se mantuvo un por ciento mayor en acuerdo con la premisa sobre: la ayuda que éstos (pares) le brindaron (60) en la premisa con valor negativo que aludía a la monopolización de la conversación por otros miembros del grupo, la muestra mostró una distribución casi equitativa de la escala (ver Tabla 3).

Tabla 3

Rol de los Pares

\begin{tabular}{lccc}
\hline \multicolumn{1}{c}{ Premisa: } & \multicolumn{3}{c}{ POR CIENTO } \\
\cline { 2 - 4 } & Acuerdo & Imparcial & Desacuerdo \\
\hline "algunas personas & & & \\
$\begin{array}{l}\text { monopolizaban } \\
\text { conversaciones en el } \\
\text { grupo de apoyo" }\end{array}$ & $30 \%$ & $40 \%$ & $30 \%$ \\
\hline Total Muestra & & & \\
\hline
\end{tabular}

Preguntas Abiertas sobre Recomendaciones y Sugerencias de los Participantes

El $95 \%$ de la muestra ofreció su opinión y sugerencias sobre el grupo de apoyo en respuesta a la parte de preguntas abiertas. Se establecieron categorías en las respuestas para tener la distribución de frecuencias y realizar el análisis.

\section{Tabla 4}

Opinión Sobre la Experiencia en el Grupo

\begin{tabular}{lc}
\hline Opiniones sobre Beneficios en: & Frecuencia \\
\hline Proceso de Salir del Clóset & 2 \\
Manejo de la Homofobia Internalizada & 2 \\
Autoconocimiento & 9 \\
Apoyo y Modelaje de Pares & 4 \\
Manejo de Relaciones de Pareja & 1 \\
Reconocimiento a las Facilitadoras & 1 \\
\hline Valor Positivo del Grupo & 4 \\
\hline
\end{tabular}


Las opiniones apuntaron a estar satisfechos con la experiencia grupal, ninguno de los participantes opinó de forma negativa sobre su experiencia. Las sugerencias para mejorar y enriquecer la experiencia de los grupos de apoyo fueron variadas (tabla 5).

Entre las sugerencias más significativas se encuentra las de aumentar la visibilidad del grupo. Esta sugerencia interfiere con la filosofía y tipo de grupo con relación a la confidencialidad y proteger a los estudiantes que permanecen en el clóset, por otro lado, también la sugerencia de hacer actividades extracurriculares y dinámicas de confraternización pueden apuntar a la misma dirección, aunque durante el semestre se organiza por lo menos una actividad, esta se realiza en ambientes controlados. Invitar recursos e invitados externos es algo que se realiza una vez por año académico, con temas variados según la necesidad del grupo, es una práctica que se lleva desde alrededor de cuatro años hasta el presente.

Tabla 5

Sugerencias para mejorar o enriquecer la experiencia grupal

\begin{tabular}{lc}
\hline Sugerencias & Frecuencia \\
\hline Aumentar la frecuencia y cantidad de horas a las & 3 \\
reuniones & 1 \\
Crear red de contactos & 3 \\
Mejorar la asistencia de los miembros & 5 \\
Estrategias para aumentar la visibilidad & 3 \\
Actividades de confraternización extracurriculares & 1 \\
Interés en facilitadores & 2 \\
Mantener facilitadores sensibles y comprometidos & 1 \\
Crear grupos de activismo & 3 \\
Permanencia del grupo & 3 \\
Integrar recursos externos & 2 \\
Mejorar las condiciones fisicas de sitio de reuniones & 1 \\
\hline Actividades dinámicas & \\
\hline
\end{tabular}

Las sugerencias de mejorar la asistencia del grupo de miembros y las condiciones físicas de las reuniones, son situaciones que no pueden controlarse por parte de las facilitadoras, la primera por el criterio de participación voluntaria y la segunda por problemas de facilidades físicas 
de nuestro Recinto y Departamento. Es importante puntualizar que esta muestra valoriza el rol de las facilitadoras y la permanencia del grupo, lo cual puede interpretarse como valores importantes y significativos para la organización, funcionamiento y mantenimiento de un grupo de apoyo para jóvenes GLB, pues el Grupo de Apoyo GLB ha atendido y atiende a una población estudiantil desprovista de servicios y aceptación.

\section{Discusión e Implicaciones para la Práctica}

Los hallazgos de esta evaluación confirman la importancia de la metodología grupal para esta población y sugieren que debe continuar el ofrecimiento de este servicio en el Departamento de Consejería para el Desarrollo Estudiantil. El DCODE fue pionero en incluir un Grupo de Apoyo para estudiantes GLB y aporta con una contribución única y sin precedentes. Se reitera el rol de ayuda y la importancia que tienen los pares a través de la dinámica grupal los cuales funcionan como sistema de apoyo a un grupo estudiantil que podría carecer de modelos de aceptación en otros ambientes sociales. Es a estos pares a quienes primero se acercan la juventud GLB para compartir el secreto de su orientación sexual en busca de una respuesta de aceptación del ambiente que les rodea. Los estudios revelan que la salida del clóset para la juventud GLB en su mayoría se inicia con pares y amigos que a su vez pueden ser también GLB y representar el inicio de la creación de un sistema de apoyo que responda a una familia (Castañeda, 1999; Mallon, 1998).

Sobre los problemas que enfrentan las personas GLB relacionados a su orientación sexual sobresale la aceptación familiar. Las relaciones familiares para la persona GLB pueden ser muy complejas, más allá de la salida del clóset, pero es este proceso el que parece tomar mayor importancia en la literatura y servicios de ayuda (Castañeda, 1999; Stewart, 1999; Mallon, 1998). La complejidad familiar puede ser una línea sugerida para posibles investigaciones en el campo del trabajo social así como a políticas y situaciones sociales que rodean a las personas GLB en nuestro país con la coyuntura actual de la discusión de la diversidad familiar. Como discutimos en apartados anteriores algunas personas se desarrollan en un ambiente familiar con modelos heterosexistas que no apoyan la realidad de la persona GLB, con posibles consecuencias en su identidad sexual por el estigma y posible marginación de ser diferente. Los 
beneficios que reconoce esta muestra ayudan al desarrollo y reconstrucción de esta identidad como lo son, poder reconocer situaciones similares entre sus pares que sirvan de modelos o alternativas al manejo de situaciones relacionadas a la orientación sexual. Reconocer que el grupo representa este espacio donde la expresión libre sin temor a juicios, aporta a redefinir la respuesta del ambiente universitario, y puede ayudar a lo que la muestra señala como otro beneficio: facilitar el ajuste a la vida universitaria.

El grupo de estudiantes mostró estar satisfechos con la figura de las facilitadoras como profesionales de ayuda lo cual reconoce el trabajo de un equipo multidisciplinario. La función de las facilitadoras parece cumplir con el objetivo de respeto y aceptación a la diversidad como cambios en respuesta del ambiente universitario que rodea a las personas que participan de la intervención grupal. Por otro lado la afirmación de recibir ayuda a través de la participación en el grupo para enfrentar problemas relacionados a la orientación sexual apoya la función y propósitos de los roles de las facilitadoras y los pares, así como las recomendaciones que añaden mantener facilitadoras sensibles y la permanencia del grupo como ayuda (las recomendaciones se discuten en el apartado anterior de hallazgos). La percepción de esta muestra sobre los beneficios que les ofreció el Grupo de Apoyo para Estudiantes GLB se interpreta como el alcance y el cumplimiento del compromiso de una filosofía de apoyo, tanto a través de las redes que surgen entre sus miembros en este tipo de grupo así, como en la función de sus profesionales de ayuda.

\section{Limitaciones y Recomendaciones}

Entre las limitaciones de este trabajo reconocemos el bajo nivel de respuesta de personas que participan o han participado del grupo de apoyo. $\mathrm{El}$ hecho de que los potenciales participantes en la investigación a quienes se contactó para invitarles fuera de inicio bajo (41 contactos) pudiera predecir el bajo nivel de respuesta. Sin embargo notamos que la mitad de las personas contactadas participó. Debe entenderse que la selección de participantes se hizo por disponibilidad y que no se intenta generalizar a todas las personas que han participado del grupo de apoyo.

Por otro lado, destacamos la poca participación de estudiantes del género femenino. La participación de estudiantes lesbianas en el Grupo no tiene un patrón sistemático pero varia a través de los semestres. Existe un rango 
de posibles explicaciones sobre la poca participación femenina discutida en la literatura, entre éstas la poca frecuencia en la búsqueda de ayuda profesional por las mujeres lesbianas, poca acceso y disponibilidad a los servicios de salud, diferencias entre género y procesos como el desarrollo de identidad sexual y salida del clóset, una doble opresión y formas híbridas de discriminación (mujer-lesbiana) entre otras, pero exponemos esta realidad como una posible línea de investigación poco estudiada en Puerto Rico y fértil para aportar con nuevos hallazgos (Ardila, 1998; Bridget, 2000; Mark, 1998).

Concientes de la realidad de un ambiente general de opresión y poco apoyo social en el que vive la juventud gay, lésbica y bisexual, la lucha por mantener sistemas de apoyo profesionales es fundamental. Los orígenes de la evaluación del modelo de Grupo de Apoyo para Estudiantes Gay, Lesbianas y Bisexuales y los resultados como hallazgos y recomendaciones se interpretan como factores positivos para el mantenimiento de esta modalidad de intervención entre los servicios del DCODE. Los grupos de apoyo para jóvenes GLB proveen espacios seguros y de aceptación que facilitan enfrentar situaciones únicas (proceso de salir del clóset, desarrollo de la identidad sexual y manejo de la homofobia, entre otros) este grupo de estudiantes durante su vida universitaria.

Nuestra recomendación apunta al ejercicio de integrar servicios dirigidos a estudiantes GLB en otros espacios universitarios para apoyar a esta minoría que carece de servicios en sus comunidades. Esta recomendación se justifica: 1) por el ambiente de rechazo social sostenido de ideologías religiosas, heterosexistas y de género que conservan un ambiente de homofobia social que afecta a todas las personas sin discriminar por orientación sexual; 2) los beneficios descritos por el estudiantado tanto en el manejo de situaciones del diario vivir relacionadas a la orientación sexual como en su autoestima; y 3) la reproducción de los problemas sociales en el micro contexto de la Universidad. Entendemos que la aportación del Modelo de Grupo de Apoyo GLB, podría utilizarse y adaptarse a las diferentes unidades del Sistema UPR y otras universidades para así contribuir a un ambiente universitario inclusivo donde no se discrimine por orientación sexual, donde todas y todos los estudiantes tengan un lugar equitativo en el espacio de la universidad y una respuesta afirmativa: la aceptación. 
Esperamos que este ejercicio de evaluación a través de la percepción y experiencia de esta muestra de estudiantes como hallazgos, generen interés en profesionales del trabajo social, consejería, psicología y otras profesiones de ayuda y relacionadas a la salud, a seleccionar tanto la metodología y modalidad de grupos de apoyo como, a la minoría GLB. Esta minoría es descrita en la literatura de las ciencias sociales como una población de alto riesgo que requiere de nuestro compromiso profesional, e invita a continuar la lucha de derechos a servicios inclusivos para todas las personas que forman el amplio espectro de la diversidad humana. Se convierte en una acción afirmativa comprometernos con las diversas formas del ser humano dónde podamos defender los principios y valores éticos que son parte fundamental de las profesiones de ayuda, y los derechos humanos de las personas con las que trabajamos, es una respuesta y acción que no podemos evadir.

\section{Referencias}

Alegre, Olga (2000). Diversidad Humana y Educación. Málaga: Ediciones Aljibe.

Ardila, Rubén (1998). Homosexualidad y Psicología. Colombia: Manual Moderno.

Bimbi, David; Grov, Christian; Nanín, José \& Parsons, Jeffrey (2006). Race, Ethnicity, Gender, and Generational Factors Associated with the Coming-Out Process Among Gay, Lesbian, and Bisexual Individuals. The Journal of Sex Research, 43,2.

Castañeda, Marina (1999). La Experiencia Homosexual: Para comprender la homosexualidad desde dentro y desde fuera. México:Paidós.

Chojnacki, Joseph \& Gelberg, Susan (1995). The Facilitation of a Gay/Lesbian/Bisexual Support-Therapy Group by Heterosexual Counselors. Journal of Counseling \& Development,73, 3, 352.

De Vidas, Michael (1999). Childhood Sexual Abuses and Domestic Violence: A Support Group for Latino Gay Men and Lesbian. 
Journal of Gay and Lesbian Social Services; Issues in Practice, Policy \& Research, 10, 2, 511.

Elze, Diane (2002). Risk factors for internalizing and externalizing problems among gay, lesbian, and bisexual adolescents. National Association of Social Worker, 26, 2.

Elze, Diane (2003). Gay, Lesbian, and Bisexual Youths' Perceptions of Their High School Environments and Comforts Schools. National Association of Social Workers, Inc.

Gay bias charge (1998). Teacher Magazine, 9, 6, 11.

Human Rights Campaign Foundation. (s.f.). Guía de Recursos para Salir del Clóset Para Personas Gays, Lesbianas, Bisexuales y Transgéneros.

López, Mabel (2003). Intervención con Clientes Gay, Lesbianas y Bisexuales. Manuscrito para uso didáctico no publicado.

Mallon, Gerald (Ed.) (1998). Foundation of Social Work Practice with Lesbian and Gay Persons. New York: The Harrington Park Press.

Mark, Frank (1998). Una Historia Natural de la Homosexualidad. Barcelona: Editorial Paidós.

Pearson, Richard (1982). A New Concept in Guidance and Counseling:Support Counseling" de Syracuse University.Taller ofrecido a Profesionales de Ayuda del Sistema de la Universidad de Puerto Rico en la Administración de Colegios Regionales.

Robinson, Kristin (1994). Addressing the Needs of Gay and Lesbian Students: The School Counselor's Role. School Counselor, 41, 5.

Rovira, Adriano (1997). Crecimiento y Desarrollo Humano. Río Piedras: Cultural Panamericana.

Russell,Sthephen \& Joyner, Kana (2001).Adolescent sexual orientation and suicide risk: Evidence from a national study. American Journal of Public Health. 91,8,1276-1281. 
Stewart, Chuck (1999). Sexually Stigmatized Communities: Reducing Heterosexism and Homophobia: An Awareness Training Manual. USA, CA.: Sage Publications.

Stewart, G. (1997). Gay and Lesbian Youth. San Diego, CA: Lucent Books.

Toseland, Ronald \& Rivas, Robert (2001). An Introduction to Group Practice. MA: Allyn \& Bacon.

Toro-Alfonso, José (2004). Las redes de apoyo social para las personas que viven con VIH/SIDA en Puerto Rico: Del aislamiento a la plena ciudadanía mediante la acción social. Revista de Ciencias Sociales, $13,28-51$.

Toro-Alfonso, José \& Varas-Díaz, Nelson (2004).Los Otros: prejuicio y distancia social hacia Gay y lesbianas en una muestra de estudiantes de nivel universitario. Revista Internacional de Psicología Clínica y de la Salud 4,3.

Van Wormer, Katherine \& McKinney, Robin (2003). What Schools Can Do To Help Gay/Lesbian/Bisexaul Youth: A Harm Reduction Approach.Adolescence, 38, 151. 
